18

\title{
Spatial Confinement of Microobjects in the Radiofrequency lon Trap in a Viscous Medium*
}

\author{
(C) A.V. Romanova, I.A. Kosternoi, and Y.V. Rozhdestvensky \\ Information Optical Technologies Center, ITMO University, \\ 197101 St. Petersburg, Russia \\ e-mail: anromanna98@gmail.com
}

Received January 18, 2020

Revised January 18, 2020

Accepted April 20, 2020

In the present article a spatial confinement of microobjects were explored in the radiofrequency Paul trap at normal pressure. Spores of Lycopodium Clavatum, $33 \mu \mathrm{m}$ in diameter, and CdSe/ZnS (core/shell) quantum dots conglomerates with size of $2-7 \mu \mathrm{m}$ were used as such microobjects. Zero-crossing orbits of these objects were observed for the first time what indicates the nonlinear nature of dynamics of these particles in localization area. Mathematical descriptions of particle dynamics in a viscous is presented. It is shown that friction value depends on the radius of microobjects and dynamic viscosity. Moreover, zero-crossing orbits of charged particles in the radiofrequency Paul trap were numerically simulated. A new method of comparative analysis of the morphology of microparticles is proposed.

Keywords: ion traps, non-linear dynamic, mass-spectrometry, biological objects.

\footnotetext{
* The 2nd international school-conference for young researchers „Smart Nanosystems for Life“, St.Petersburg, Russia, December 10-13, 2019.

Полный текст статьи опубликован в „Оptics and Spectroscopy“ 2020 V. 128. N 8.
} 